\title{
ОПЫТ ПРИМЕНЕНИЯ СИНТЕТИЧЕСКИХ ПРОТЕЗОВ В ХИРУРГИИ ПРОЛАПСА ЖЕНСКИХ ПОЛОВЫХ ОРГАНОВ
}

\author{
Н. Ф. Захаренко ${ }^{1}$, Н. В. Косей ${ }^{1}$, Н. Ю. Педаченко ${ }^{2}$ \\ 'Институт педиатрии, акушерства и гинекологии НАМН Украины, \\ ${ }^{2}$ Национальная медицинская академия последипломного образования имени П. Л. Шупика МЗ Украины, г. Киев

\section{EXPERIENCE OF APPLICATION OF SYNTHETIC PROSTHESIS IN SURGERY OF THE WOMEN'S GENITALS PROLAPSE}

\author{
N. F. Zakharenko, N. V. Kosey ${ }^{1}$, N. Yu. Pedachenko ${ }^{2}$ \\ ${ }^{1}$ Institute of Pediatry, Obstetrics and Gynecology, \\ ${ }^{2}$ Shupyk National Medical Academy of Postgraduate Education, Kiev
}

\section{Рефрерат}

Цель. Оптимизация хирургического лечения пролапса женских половых органов (ЖПО).

Материалы и методы. Проанализирован десятилетний опыт выполнения реконструктивных операций на влагалище с использованием синтетических сетчатых протезов из пролена Gynemesh Soft (слингов) у 194 женщин в возрасте от 42 до 76 лет по поводу пролапса ЖПО.

Результаты. Влагалищная экстирпация матки выполнена у $93(47,9 \%)$ женщин, по поводу полного выпадения матки - у 43 (22,2\%), лейомиомы матки - у 26 (13,4\%), рецидивирующих декубитальных язв - у 9 (4,6\%), патологии шейки матки - у 9 (4,6\%), гиперпластических процессов в эндометрии в период менопаузы, подтвержденных по результатам гистологического исследования - у 6 (3,1\%). У 101 (52,1\%) пациентки проведено хирургическое лечение пролапса ЖПО с использованием синтетических слингов без предварительной гистерэктомии.

Обсуждение. Проанализированы типичные осложнения хирургического вмешательства. По данным активного мониторинга, осложнения выявлены у 5,2\% пациенток, в том числе у 8 (4,1\%) - эрозия влагалища после установки систем Prolift posterior, y $1(0,5 \%)$ - пролапс протеза в мочевой пузырь после применения системы Prolift anterior, у 1 (0,5\%) - рецидив пролапса после установки системы Prolift total.

Выводы. Профилактикой осложнений является точное соблюдение технологии установки имплантата с соблюдением рекомендаций: соответствие площади сетки площади пролабирующей слизистой оболочки, отсутствие натяжения при установке протеза, расположение протеза субфасциально, фиксация сетки в целях предупреждения ее смещения, назначение адекватной адъювантной терапии после операции.

Ключевые слова: пролапс женских половых органов; осложнения; синтетические сетчатые протезы; рецидивы.

Abstract

Objective. Optimization of surgical treatment for the women's genitals prolapse.

Materials and methods. Ten-year experience of reconstructive vaginal operations, using synthetic net prosthesis, manufactured from prolene Gynemesh Soft (slings), in 194 women, ageing $42-76$ yrs for the women's genitals prolapse, was aalysed.

Results. Vaginal extirpation of uterus was performed in $93(47.9 \%)$ women, for total prolapses uteri - in $43(22.2 \%)$, for leiomyoma uteri - in $26(13.4 \%)$, recurrent decubital ulcers - in $9(4.6 \%)$, pathology of cervix uteri - in $9(4.6 \%)$, hyperplastic processes in endometrium in menopause period, confirmed by results of histological investigation - in $6(3.1 \%)$. In $101(52.1 \%)$ women-patients surgical treatment of the genitals prolapse, using synthetic slings without previous hysterectomy, was conducted.

Discussion. Typical complications of surgical intervention were analyzed. In accordance to the active monitoring data, complications were revealed in $5.2 \%$ of women-patients, including in $8(4.1 \%)$ - vaginal erosion after installation of the Prolift posterior system, in 1 $(0.5 \%)$ - a prosthesis prolapse into bladder after application of the Prolift anterior system, in $1(0.5 \%)$ - a prolapse recurrence after the Prolift total installation.

Conclusion. For prophylaxis of complications the implant installation technology must be precisely followed in accordance to actual recommendations: conformity between the net area and the bulging mucosa area, absence of tension while prosthesis installation, subfascial position of prosthesis, the net fixation for its replacement prophylaxis, administration of adequate adjuvant therapy postoperatively.

Keywords: prolapse of genitals; complications; synthetic net prosthesis; recurrences.

Пролапс ЖПО выявляют у 40\% женщин в возрасте от 30 до 45 лет, в возрасте старше 50 лет - у 50\% [1]. В структуре хирургических вмешательств в гинекологической практике операции коррекции выпадения матки и влагалища занимают третье место после вмешательств по пово- ду доброкачественных новообразований и эндометриоза $[1,2]$. Согласно данным Американской ассоциации урологов, хирургическое лечение пролапса ЖПО и/или стрессового недержания мочи показано 11\% женщин [1, 2], у 50\% женщин старше 60 лет выявляют различные формы пролапса внутренних жПО [3]. Общее число женщин, у которых диагностируют пролапс ЖПО, в США и странах Европы составляет 36 млн. [4].

В нашей стране, по данным литературы, пролапс ЖПО выявляют у 15 - 40\% гинекологических больных 
[5 - 7]. Одной из основных проблем при пролапсе ЖПО является недержание мочи, по данным зарубежных исследователей [2,8 - 10], частота его достигает 45\%. Эффективность хирургического лечения пролапса ЖПО с использованием синтетических материалов может достигать 81 - 100\% [8, 9]. Одной из предпосылок пролапса ЖПО является дисплазия соединительной ткани, поэтому использование синтетических слингов является методом выбора с уровнем успешности до 84\% после 5 лет наблюдения [10]. Примерно 90\% вмешательств по поводу всех вариантов пролапса ЖПО и недержания мочи в США в 2009 г. были выполнены с использованием миниинвазивных технологий. Несмотря на высокие показатели успеха, у 5 - 20\% пациенток при применении слингов лечение оказалось неэффективным, возник повторный пролапс органов таза и влагалища [11]. Широкое распространение и высокая частота выполнения операций обусловили увеличение частоты неудач лечения и потребности в соответствующих методах реабилитации.

Проблема безопасности использования синтетических имплантатов актуальна и в настоящее время [9, 12, 13]. Несвоевременное выявление осложнений может стать причиной летального исхода, повторных хирургических вмешательств, существенно ухудшить прогноз, обусловить инвалидизацию пациентОК.

Консенсус относительно управления стойким или рецидивирующим стрессовым недержанием мочи после неудачного применения синтетического протеза не достигнут. При возникновении осложнений многие женщины и хирурги предпочитают избегать повторной процедуры и выбирают лоскут аутологичной ткани. Для оценки эффективности применения сетчатых имплантатов нами проведено эмпирическое исследование.

\section{МАТЕРИАЛЫ И МЕТОДЫ ИССЛЕДОВАНИЯ}

С ноября 2007 г. на базе гинекологического отделения КГКБ № 9 у 194 пациенток выполнены влага- лищные реконструктивные операции с использованием синтетических сетчатых протезов из пролена Gynemesh Soft (Johnson \& Johnson, Швейцария), из них с использованием систем Prolift posterior - у 121, Prolift anterior - y 63, Prolift total - y 10, в зависимости от особенностей пролапса ЖПО. Системы представляют собой сетчатый имплантат для хирургического лечения рецидивирующего опущения стенок влагалища, который состоит из рассасывающегося моноволокна полиглекапрона-25 и нерассасывающихся волокон полипропилена. После имплантации сетки нити полиглекапрона-25 практически полностью рассасываются через 3 - 4 мес [4, 8].

Особенностью таких сетчатых имплантатов является их адаптация при различных видах пролапса ЖПО, наличие выкроенных готовых сеток и необходимое число трубчатых проводников. Показанием к установке сетчатого имплантата для реконструкции переднего отдела тазового дна (Prolift ${ }^{\mathrm{TM}}$ anterior) является опущение передней стенки влагалища с формированием цистоуретроцеле III - IV степени; реконструкции заднего отдела тазового дна (Prolift ${ }^{\mathrm{TM}}$ posterior) - опущение задней стенки влагалища с формированием энтеро- и ректоцеле II III степени [8]. Опущение передней и задней стенок влагалища II - III степени, полное выпадение матки и стенок влагалища являются показанием к установке сетчатого имплантата для полной реконструкции тазового дна (Prolift ${ }^{\mathrm{TM}}$ total).

Операции по установке имплантатов не требуют широкого иссечения клетчатки забрюшинного пространства, необходимы лишь проведение небольших каналов вдоль кардинальных связок по направлению к седалищной ости и фиксация «ножек» протеза в области крестцово-остистой связки при установке Prolift posterior. При использовании Prolift anterior сетку размещают субфасциально под мочевым пузырем, «ножки» протеза фиксируют в области запирательных отверстий. Имплантат устанавливают без натяжения и фиксируют рассасывающейся нитью $[8,14]$.

\section{РЕЗУЛЬТАТЫ}

При хирургическом лечении пролапса ЖПО влагалищная экстирпация матки произведена 93 (47,9\%) женщинам, в том числе по поводу полного выпадения матки - 43 $(22,2 \%)$, лейомиомы матки - 26 (13,4\%), рецидивирующих декубитальных язв - 9 (4,6\%), патологии шейки матки - 9 (4,6\%), гиперпластических процессов в эндометрии в период менопаузы, подтвержденных результатами гистологического исследования - 6 (3,1\%). У 101 (52,1\%) пациентки проведено хирургическое лечение пролапса с использованием синтетических слингов без предварительной гистерэктомии.

Оперативное вмешательство выполняли под спинальной анестезией. Длительность операции от 45 до 120 мин, в зависимости от объема операции (с экстирпацией матки или без нее).

Интраоперационных осложнений не было. Кровопотеря во время операции составляла 150 - 200 мл, до 300 мл - при выполнении гистерэктомии. Продолжительность лечения больных в стационаре от 4 до 10 сут, в среднем 8 сут. Течение послеоперационного периода без осложнений, всем пациенткам проведена санация влагалища с использованием свечей с бетадином, антибактериальная терапия.

В сроки до 10 лет активного наблюдения после оперативного лечения удовлетворительные результаты по стабильной постхирургической коррекции пролапса ЖПО достигнуты у 184 женщин.

\section{ОБСУЖДЕНИЕ}

По данным активного мониторинга у 5,2\% пациенток в дальнейшем возникли осложнения, в том числе у 8 (4,1\%) - эрозия влагалища после установки систем Prolift posterior, y 1 (0,5\%) - пролапс протеза в мочевой пузырь после применения системы Prolift anterior, у 1 (0,5\%) - рецидив пролапса ЖПО после установки системы Prolift total.

Приводим наблюдения. 1. Пациентка И., 45 лет, госпитализирована с диагнозом: опущение задней стенки влагалища, ректоцеле. 
Осуществлена пластика задней стенки влагалища с использованием синтетического протеза Prolift posterior. Течение послеоперационного периода без осложнений, пациентка выписана на 4-е сутки. При активном осмотре через 1 мес патология не выявлена. Через 6 мес жалобы на появление выделений из половых путей с примесью крови. При гинекологическом осмотре обнаружена эрозия задней стенки влагалища в месте смещения протеза со стороны входа во влагалище.

Назначена консервативная терапия: свечи Бетадин, кольпотрофин.

Через 1 мес произведена операция: иссечен участок синтетического протеза, зашита слизистая оболочка влагалища. При осмотре через 3 мес жалоб нет, дефекты слизистой оболочки, пролабирование протеза не обнаружены.

Через 5 лет у пациентки обнаружен участок эрозии сетчатого протеза диаметром до 4 мм. Рекомендовано: свечи Депантол №10, кольпотрофин через день постоянно. Через 2 года пациентка осмотрена, состояние удовлетворительное, сетка смещена, прощупывается при пальпации. Визуализируется эрозия диаметром до 4 мм.

Возможные причины осложнения: неудовлетворительная фиксация протеза, использование для фиксации рассасывающейся нити.

2. Пациентка Л., 52 лет, госпитализирована с диагнозом: опущение стенок влагалища, ректоцеле, цистоцеле, сахарный диабет II типа. Выполнены экстирпация матки без придатков, пластика влагалища с использованием синтетического протеза Prolift total. Течение послеоперационного периода без осложнений, пациентка выписана на 7-е сутки.

Осмотрена через 1 мес, состояние удовлетворительное. Через 2 мес появились жалобы на обильные выделения из половых путей с примесью крови. По данным гинекологического осмотра обнаружена әрозия задней стенки влагалища в месте расположения сетки, гипергрануляционный полип.

Назначены санация влагалища, свечи Гексикон. Произведено уда- ление полипа с иссечением участка протеза и зашиванием слизистой оболочки. Рекомендовано местно применение свечей Бетадин № 10 , затем кольпотрофин через день постоянно.

Осмотрена через 1 мес, әрозия сетки не обнаружена, рекомендовано продолжить применение кольпотрофина 2 раза в неделю.

При осмотре через 5 мес выявлена эрозия сетки на протяжении $2-3$ мм. Предложено иссечение сетки, от которого пациентка отказалась. Назначены местно свечи Депантол в течение 10 сут, затем кольпотрофин 2 раза в неделю. Через 3 мес при осмотре состояние пациентки удовлетворительное, жалоб нет. По данным гинекологического осмотра участок эрозии визуализируется только с помощью кольпоскопа. Предложено продолжить введение кольпотрофина. Через 4 года во время осмотра состояние без изменений, через 2 года - состояние прежнее, пациентка жалоб не предъявляет.

3. Пациентка С., 48 лет, госпитализирована с диагнозом: опущение стенок влагалища, цистоцеле, ректоцеле, дисплазия шейки матки.

Выполнены влагалищная экстирпация матки без придатков, пластика передней и задней стенок влагалища с использованием синтетического протеза Prolift total. Течение послеоперационного периода без осложнений, пациентка выписана на 7-е сутки. Рекомендовано: свечи Бетадин на протяжении 10 сут с последующим использованием кольпотрофина 2 раза в неделю постоянно.

Осмотрена через 1 мес, без особенностей. Через 2 мес появились жалобы на обильные выделения из половых путей с примесью крови. Обнаружена эрозия на передней стенке влагалища, назначена санация влагалища. Через 1 мес произведено иссечение участка сетчатого протеза с зашиванием слизистой оболочки. Назначены свечи Бетадин, кольпотрофин.

Пациентка приглашена на осмотр через 6 мес, эрозия не выявлена. При активном мониторинге через 8 лет эрозия сетки не обнаружена. Предполагаемая причина осложнения - чрезмерное натяжение сетки при установлении Prolift anterior.

4. Пациентка Г., 54 лет, госпитализирована с диагнозом: опущение стенок влагалища, цистоцеле, ректоцеле, стрессовое недержание мочи. Заинтересована в сохранении матки. Выполнены пластика передней и задней стенок влагалища с использованием синтетического протеза Prolift anterior и Prolift posterior, ypeтропексия с использованием TVT-O. Течение послеоперационного периода без осложнений, пациентка выписана на 8-е сутки.

При гинекологическом осмотре через 2 и 6 мес патология не выявлена, расположение сетки правильное, болезненности при пальпации нет, моча удерживается.

Через 10 мес пациентка обратилась с жалобами на учащенное мочеиспускание, боль при мочеиспускании, а также боль при ходьбе, отдающую в мочевой пузырь. При влагалищном осмотре отмечена резкая болезненность переднего свода влагалища, больше слева, в месте расположения протеза ощущается натяжение участка сетки Prolift anterior. При УЗИ мочевого пузыря в просвете визуализируется гиперэхогенный тяж слева.

Проведена цистоскопия, обнаружен пролапс сетки в мочевой пузырь со стороны влагалища с множественными микролитами на ней. Рекомендовано оперативное лечение. Произведено иссечение участка протеза, пролабирующего в стенку мочевого пузыря со стороны влагалища, зашивание стенки мочевого пузыря с последующим зашиванием стенки влагалища. После операции в пузыре оставлен катетер Фолея на 10 сут. Пациентка выписана на 12-е сутки с рекомендациями продолжить местную противовоспалительную терапию, а также местную гормональную терапию для улучшения репаративных процессов слизистой оболочки.

Осмотрена через 3 мес. Жалобы на учащенное мочеиспускание (через каждые 2 - 3 ч). При осмотре влагалища патологических изменений нет. Проведена цистоскопия: устья мочеточников свободные, выброс мочи не затруднен, слизистая 
оболочка мочевого пузыря розовая, без дефекта.

Через 6 мес пациентка приглашена на осмотр. Жалоб нет. Может удерживать мочу до 4 ч. Слизистая оболочка влагалища розовая, своды сформированы. Зона расположения Prolift anterior чувствительна, задняя стенка влагалища безболезненна. При ректальном осмотре сетка расположена адекватно, не смещена. Рекомендовано продолжить местную гормональную терапию.

Через 3 года осмотрена в плановом порядке, сетка расположена адекватно, смещений и эрозий нет. Рекомендовано продолжить применение кольпотрофина.

По данным анализа осложнений при использовании синтетических слингов для коррекции пролапса ЖПО, для предупреждения эрозий необходимо соблюдать такие условия при установке сетчатых имплантатов:

- соответствие площади сетки площади пролабирующей слизистой оболочки;

- недопущение натяжения при установке протеза;

- расположение протеза субфасциально;

- фиксация сетки в целях предупреждения ее смещения;

- назначение адекватной адъювантной терапии после операции.

Накопленный нами опыт свидетельствует о необходимости более тщательного отбора пациенток для установки синтетических слингов и взвешенного отношения к повсеместному использованию mesh-материалов. Несмотря на высокую эффективность, операция может сопровождаться осложнениями. Для профилактики осложнений чресвлагалищной кольпопексии с использованием синтетических протезов необходим строгий отбор пациенток, хорошее знание топографической анатомии малого таза и промежности, точное соблюдение технологии установки имплантата с соблюдением рекомендаций, адекватная терапия в послеоперационном периоде.

\section{REFERENCES}

1. Vdovichenko JuP, Grigorenko AP, Bojchuk VM, ta in. Dejaki aspekti patogenezu vipadinnja matki ta stinok pihvi u zhinok, hirurgichna reabilitacija. Reproduktivnoe zdorov'e zhenshhiny. 2005;1(26):198-9. [In Ukrainian].

2. Olsen AL, Smith VJ, Bergstrom JO, et al. Epidemiology of surgically managed pelvic organ prolapse and urinary incontinence. Obstet Gynecol. 1997;89:501-6.

3. Slobodjanjuk BA. Sravnitelnyj analiz laparoskopicheskogo i vaginal'nogo dostupov pri lechenii genital'nogo prolapsa s ispolzovaniem sinteticheskih materialov [dissertation]. Moskva, 2009. [In Russian].

4. The US Food and Drug Administration. FDA Public Health Notification: Serious Complications Associated with Transvaginal Placement of Surgical Mesh in Repair of Pelvic Organ Prolapse and Stress Urinary Incontinence. Available from: www.fda.gov/cdrh/ safety/102008-surgicalmesh.html. Accessed February 23,2009115. Trowbridge.

5. Strizhakova MA. Anomalii polozhenija polovyh organov. Klinicheskie lekcii po akusherstvu i ginekologii. Moskva: Medicina; 2004:327339. [In Russian].

6. Chajka VK, Barkalov OA, Trifonova EF, Zheleznaja AA. Aktual'nye mediko-social'nye voprosy uroginekologii. Mediko-social'nye problemy sem'i. 2001;6(4):74-80. [In Russian].

7. Tatarchuk TF, Efimenko OA, Islamova AO. Menopauza: novyj vzgljad na staruju problemu. Al'manah reproduktivnogo zdorov'ja. 2013:106-14. [In Russian].
8. Ali S, Han HC, Lee LC. A prospective randomized trial using Gynemesh PS for the repair of anterior vaginal wall prolapse. Int Urogynecol J. 2006;17, suppl. 2:221.

9. Butrick CW. Do guns kill people or...? The mesh dilemma. Ibid. 2010;21:133-4.

10. Feiner B, Jelovsek JE, Maher C. Efficacy and safety of transvaginal mesh kits in the treatment of prolapse of the vaginal apex: a systematic review. BJOG. 2009;116, suppl.1:15-24.

11. Miller D1, Lucente V, Babin E, Beach P, Jones P, Robinson D. Prospective clinical assessment of the transvaginal mesh technique for treatment of pelvic organ prolapse-5-year results. Female Pelvic Med Reconstr Surg. 2011 May;17(3):139-43.

12. Abramjan KN. Profilaktika i lechenie oslozhnenij jekstraperitoneal'noj vaginopeksii (operacii Prolift): [thesis]. Moskva; 2011. 28 p. [In Russian].

13. Gluhov EJu, Plotko EJe, Mamin JeL, Hajutin VN. Opyt primenenija sinteticheskih materialov $v$ hirurgii genital'nogo prolapsa i stressovogo nederzhanija mochi. Rossijskij vestnik akushera i ginekologa. Moskva, 2008; Specvypusk: 14-5. [In Russian].

14. Maher C, Baessler K, Glazener CM, Adams EJ, Hagen S. Surgical management of pelvic organ prolapse in women: a short version Cochrane review. Neurourol Urodyn. 2008;27(1):3-12. 